\title{
Adjuvant treatment of HER2-positive breast cancer: winning efforts continue to improve HER2-positive patient outcome long-term
}

\author{
Rachel C Jankowitz and Adam M Brufsky*
}

\begin{abstract}
Randomized adjuvant trials continue to show significant reductions in distant recurrence and death for early-stage women treated with adjuvant trastuzumab. BCIRG-006 showed superior disease-free and overall survival of two trastuzumab-containing regimens in comparison to a non-trastuzumabcontaining regimen. The rates of disease-free and overall survival were not statistically different for the two trastuzumab-containing arms. Ongoing study is needed to identify markers of resistance to trastuzumab and incorporate newer agents in the adjuvant setting in order to further decrease rates of distant recurrence and death from HER2-positive breast cancer.
\end{abstract}

Approximately $20 \%$ of breast cancers exhibit amplification of the HER2 proto-oncogene on chromosome 17, and without adequate treatment these tumors tend to have an aggressive natural history [1,2]. Large randomized adjuvant trials show significant reductions in distant recurrence and death for early-stage women treated with adjuvant trastuzumab [3-6]. Recent long-term follow-up of the joint analysis of the NCCTG 9831 and B-31 studies show improved disease-free survival (DFS) and overall survival (OS) for women who received adjuvant trastuzumab [3], with a trend toward greater improvement in DFS with concurrent use of trastuzumab and chemotherapy in comparison to sequential use of trastuzumab after chemotherapy in NCCTG 9831 [7]. These groundbreaking results were tempered by a relatively high rate of clinically significant congestive heart failure (2.5\%) with adjuvant regimens containing both an anthracycline

*Correspondence: brufskyam@upmc.edu

University of Pittsburgh Cancer Institute, Magee-Women's Hospital, 300 Halket Street, Suite 4628, Pittsburgh, PA 15213, USA and traztuzumab $[3,4]$. It is with great interest, therefore, that Dr Slamon and colleagues [5] published the longawaited results of the pivotal Breast Cancer International Research Group 006 (BCIRG-006) trial, containing a non-anthracycline-containing anti-Her2 adjuvant chemotherapy regimen in one arm, in the New England Journal of Medicine in October 2011.

BCIRG-006 included over 3,000 women with either high-risk node-negative or node-positive early-stage HER2-positive breast cancer. HER2 status was centrally determined. Women were randomized to one of three regimens: (1) docetaxel plus carboplatin for six cycles concurrently with trastuzumab followed by an additional 34 weeks of trastuzumab ( $\mathrm{TCH})$; (2) doxorubicin and cyclophosphamide for four cycles followed by docetaxel for four cycles with trastuzumab starting with docetaxel and continuing for one year (AC-TH); or (3) a regimen of doxorubicin and cyclophosphamide for four cycles followed by docetaxel for four cycles (AC-T). At median follow-up of 65 months, the two trastuzumab-containing arms (TCH and $\mathrm{AC}-\mathrm{TH}$ ) showed statistically significant improvements in both DFS (AC-TH 84\%, TCH $81 \%$ versus $\mathrm{AC}-\mathrm{T} 75 \%, P<0.001$ ) and $\mathrm{OS}(\mathrm{AC}-\mathrm{TH} 92 \%, \mathrm{TCH}$ $91 \%$ versus $A C-T$ $87 \%, P<0.001)$ in comparison to the non-trastuzumab-containing arm (AC-T). The rates of DFS and OS were not statistically different for the two trastuzumab-containing arms, but the study was not powered to detect equivalence between these two regimens. The benefit of both $\mathrm{TCH}$ and $\mathrm{AC}-\mathrm{TH}$ over $\mathrm{AC}-\mathrm{T}$ was confirmed in patients with lymph node-negative disease, patients with lymph node-positive disease, and patients with four or more lymph node-positive disease.

An analysis of topoisomerase II (TOP2A) gene amplification and DFS was also performed. In the $35 \%$ of women with HER2-positive breast cancer that co-amplified HER2 and TOP2A on chromosome 17, there appeared to be no incremental benefit to traztuzumab in the $\mathrm{TCH}$ and $\mathrm{AC}-\mathrm{TH}$ arms over $\mathrm{AC}-\mathrm{T}$.

A five-fold higher rate of congestive heart failure was seen with AC-T plus trastuzumab than with $\mathrm{TCH}(2.0 \%$ and $0.4 \%$, respectively, $P<0.001$ ), and more acute 
leukemias were seen in the two anthracycline-containing arms than in the $\mathrm{TCH}$ arm. Vomiting, arthralgias, myalgias, neuropathy, neutropenia and leukopenia were significantly lower in the TCH group; anemia and thrombocytopenia were lower in the $\mathrm{AC}-\mathrm{TH}$ group, and there was no significant difference in febrile neutropenia between the arms.

There were fewer distant recurrences of breast cancer in the $\mathrm{AC}-\mathrm{TH}$ arm than the $\mathrm{TCH}$ arm (124 versus 144). There were more congestive heart failure events in the AC-TH arm (21 versus 4), and one acute leukemia in both arms, for a total event rate of 146 for AC-TH versus 149 for TCH.

In summary, there are good reasons to administer either $\mathrm{TCH}$ or an anthracycline-containing regimen (AC$\mathrm{TH}$ or AC-paclitaxel/traztuzumab) in the adjuvant setting after local therapy for HER2-positive early stage breast cancer. It is gratifying that both regimens have a 5 year OS rate above $90 \%$, and that both regimens have a 5 year DFS rate of at least $73 \%$ in women with poor risk disease with four or more positive lymph nodes. The natural history of this aggressive subtype of breast cancer has indeed been changed. Which regimen chosen will likely depend on the comorbidity of the individual patient, and the desire to avoid cardiotoxicity.

Looking forward, no clear marker has materialized as a reliable predictor of traztuzumab resistance in the adjuvant setting. Newer agents are under active investigation and may improve outcomes for early-stage patients in combination with traztuzumab-based adjuvant therapy. There also remains a lack of clarity regarding traztuzumab benefit for HER2-negative and/or HER2-low patients. Finally, traztuzumab has yielded major advances in the treatment of an aggressive breast cancer phenotype, but oncologists remain divided regarding the relative riskbenefit ratio of anthracyclines within traztuzumabcontaining adjuvant regimens $[8,9]$.

Mechanisms of resistance to trastuzumab are complex and likely involve, in part, alternative signaling pathways and/or constitutive activation of the phosphoinositide 3-kinase/Akt signaling pathway. Newer agents show promise in resistant patients with advanced disease. Pertuzumab, a monoclonal antibody that binds to HER2 and inhibits heterodimerization with other receptors of the Her superfamily, has shown progression-free survival benefit in a phase III study in advanced disease [10] and is being studied in combination with trastuzumab with either anthracyline or non-anthracycline-based chemotherapy in the ongoing adjuvant APHINITY trial (NCT01358877). Trastuzumab-DM1, a conjugate of trastuzumab and a potent microtubule polymerization inhibitor (a derivate of maytansine) has shown a progression-free survival advantage in a phase II study of advanced disease [11]. Persistent HER3 signaling is another mechanism of therapeutic resistance, and antiHER3 antibodies are in pre-clinical development. Combination studies of HER2-targeted agents with more potent, irreversible inhibitors of the epidermal growth factor receptor-HER2 family, such as Neratinib, are ongoing (NCT01423123). Retrospective analyses showing trastuzumab benefit in HER2-negative patients [12] and a recent revised analysis of the N9831 trial showed similar DFS improvements with trastuzumab therapy in patients HER2-positive by US Food and Drug Administration criteria, but negative by the revised American Society of Clinical Oncology (ASCO)/College of American Pathologists (CAP) criteria [13], making the ongoing prospective NSABP trial (NCT01275677) looking at trastuzumab in HER2-low patients a priority to define trastuzumab benefit for those patients considered HER2low or -negative.

Despite improvements in treatment of advanced HER2-positive disease, many patients ultimately die due to progression of disease at visceral sites, so it is critical to increase success rates in the adjuvant setting.

\section{Abbreviations}

BCIRG-006, Breast Cancer International Research Group 006; DFS, disease-free survival; OS, overall survival.

\section{Competing interests}

The authors declare that they have no competing interests.

Published: 30 April 2012

\section{References}

1. Wolff AC, Hammond ME, Schwartz JN, Hagerty KL, Allred DC, Cote RJ, Dowsett M, Fitzgibbons PL, Hanna WM, Langer A, McShane LM, Paik S, Pegram MD, Perez EA, Press MF, Rhodes A, Sturgeon C, Taube SE, Tubbs R, Vance GH, van de Vijver M, Wheeler TM, Hayes DF; American Society of Clinical Oncology; College of American Pathologists: American Society of Clinical Oncology/College of American Pathologists guideline recommendations for human epidermal growth factor receptor 2 testing in breast cancer. J Clin Oncol 2007, 25:118-145.

2. Dawood S, Broglio K, Buzdar AU, Hortobagyi GN, Giordano SH: Prognosis of women with metastatic breast cancer by HER2 status and trastuzumab treatment: an institutional-based review. J Clin Oncol 2010, 28:92-98.

3. Perez EA, Romond EH, Suman VJ, Jeong JH, Davidson NE, Geyer CE Jr, Martino S, Mamounas EP, Kaufman PA, Wolmark N: Four-year follow-up of trastuzumab plus adjuvant chemotherapy for operable human epidermal growth factor receptor 2-positive breast cancer: joint analysis of data from NCCTG N9831 and NSABP B-31. J Clin Oncol 2011, 29:3366-3373.

4. Piccart-Gebhart MJ, Procter M, Leyland-Jones B, Goldhirsch A, Untch M, Smith I, Gianni L, Baselga J, Bell R, Jackisch C, Cameron D, Dowsett M, Barrios CH, Steger G, Huang CS, Andersson M, Inbar M, Lichinitser M, Láng I, Nitz U, Iwata H, Thomssen C, Lohrisch C, Suter TM, Rüschoff J, Suto T, Greatorex V, Ward C, Straehle C, McFadden E, et al:: Trastuzumab after adjuvant chemotherapy in HER2-positive breast cancer. N Engl J Med 2005, 353:1659-1672.

5. Slamon D, Eiermann W, Robert N, Pienkowski T, Martin M, Press M, Mackey J, Glaspy J, Chan A, Pawlicki M, Pinter T, Valero V, Liu MC, Sauter G, von Minckwitz G, Visco F, Bee V, Buyse M, Bendahmane B, Tabah-Fisch I, Lindsay MA, Riva A, Crown J; Breast Cancer International Research Group: Adjuvant trastuzumab in HER2-positive breast cancer. N Engl J Med 2011 365:1273-1283.

6. Joensuu H, Kellokumpu-Lehtinen PL, Bono P, Alanko T, Kataja V, Asola R, Utriainen T, Kokko R, Hemminki A, Tarkkanen M, Turpeenniemi-Hujanen T, Jyrkkiö S, Flander M, Helle L, Ingalsuo S, Johansson K, Jääskeläinen AS, Pajunen M, Rauhala M, Kaleva-Kerola J, Salminen T, Leinonen M, Elomaa I, 
Isola J; FinHer Study Investigators: Adjuvant docetaxel or vinorelbine with or without trastuzumab for breast cancer. N Engl J Med 2006, 354:809-820.

7. Perez EA, Suman VJ, Davidson NE, Gralow JR, Kaufman PA, Visscher DW, Chen B, Ingle JN, Dakhil SR, Zujewski J, Moreno-Aspitia A, Pisansky TM, Jenkins RB: Sequential versus concurrent trastuzumab in adjuvant chemotherapy for breast cancer. J Clin Oncol 2011, 29:4491-4497.

8. Rocque G, Onitilo A, Engel J, Pettke E, Boshoven A, Kim K, Rishi S, Waack B, Wisinski KB, Tevaarwerk A, Burkard ME: Adjuvant therapy for HER2+ breast cancer: practice, perception, and toxicity. Breast Cancer Res Treat 2012, 131:713-721.

9. Press MF, Sauter G, Buyse M, Bernstein L, Guzman R, Santiago A, Villalobos IE, Eiermann W, Pienkowski T, Martin M, Robert N, Crown J, Bee V, Taupin H, Flom KJ, Tabah-Fisch I, Pauletti G, Lindsay MA, Riva A, Slamon DJ: Alteration of topoisomerase Il-alpha gene in human breast cancer: association with responsiveness to anthracycline-based chemotherapy. J Clin Oncol 2011, 29:859-867.

10. Baselga J, Cortés J, Kim SB, Im SA, Hegg R, Im YH, Roman L, Pedrini JL, Pienkowski T, Knott A, Clark E, Benyunes MC, Ross G, Swain SM; CLEOPATRA Study Group: Pertuzumab plus trastuzumab plus docetaxel for metastatic breast cancer. N Engl J Med 2012, 366:109-119.
11. Hurvitz S, Dirix L, Kocsis J, Gianni L, Lu J, Vinholes J, Song C, Tong B, Chu YW, Perez EA: Trastuzumab emtansine (T-DM1) vs trastuzumab plus docetaxel $(\mathrm{H}+\mathrm{T})$ in previously-untreated HER2-positive metastatic breast cancer (MBC): primary results of a randomized, multicenter, open-label phase II study (TDM4450g/B021976) [abstract 5001]. Eur J Cancer 2011, 47:S330.

12. Paik S, Kim C, Wolmark N: HER2 status and benefit from adjuvant trastuzumab in breast cancer. NEng/ J Med 2008, 358:1409-1411.

13. Perez EA, Dueck AC, McCullough AE, Reinholz MM, Tenner KS, Davidson NE, Gralow J, Harris LN, Kutteh LA, Hillman DW, Jenkins RB, Chen B: Predictability of adjuvant trastuzumab benefit in N9831 patients using the ASCO/CAP HER2-positivity criteria. J Natl Cancer Inst 2012, 104:159-162.

doi:10.1186/bcr3120

Cite this article as: Jankowitz RC, Brufsky AM: Adjuvant treatment of HER2positive breast cancer: winning efforts continue to improve HER2-positive patient outcome long-term. Breast Cancer Research 2012, 14:308. 